\title{
Pacientes con cáncer de recto localmente avanzado en tratamiento neoadyuvante con quimiorradioterapia en el Instituto Nacional de Cancerología en 2010
}

\author{
Giovanna Rivas ${ }^{1,2}$, Fabio Olivella Cicero ${ }^{1,2}$, José Alexander Carreño3 ${ }^{3}$ Viviana Rodríguez ${ }^{4}$
}

1 Grupo de Oncología Clínica, Instituto Nacional de Cancerología, ESE, Bogotá (Colombia).
2 Universidad El Bosque, Bogotá (Colombia).
${ }^{3}$ Grupo de Investigación Clínica, Instituto Nacional de Cancerología, ESE, Bogotá (Colombia).
${ }^{4}$ Grupo de Epidemiología Clínica, Universidad El Bosque, Bogotá (Colombia).

Objetivos. Describir las características sociodemográficas y clínicas de un grupo de pacientes con cáncer de recto localmente avanzado tratados con quimiorradioterapia neoadyuvante, y realizar un diagnóstico situacional del manejo.

Materiales y métodos. Se efectuó un estudio tipo series de casos, con la información clínica de 41 pacientes atendidos durante 2010 en el Instituto Nacional de Cancerología.

Resultados. La edad promedio fue 61 años, con un $61 \%$ de hombres; el estado funcional por Karnofsky fue igual o mayor al $70 \%$. El esquema neoadyuvante más utilizado fue 5 -fluorouracilo y leucovorín en bolos concomitante con radioterapia; 30 pacientes completaron el tratamiento con buena tolerancia, 21 fueron llevados a cirugía con preservación del esfínter en 10 de ellos, 18 lograron respuesta patológica; 14, parcial; y 4, completa.

Conclusión. Los pacientes con cáncer de recto localmente avanzado que recibieron tratamiento neoadyuvante con quimiorradioterapia en el Instituto Nacional de Cancerología en 2010 en su mayoría fueron hombres con buen estado funcional; el principal esquema de quimioterapia empleado fue 5-fluorouracilo y leucovorín en bolos, logrando resecabilidad del tumor, preservación del esfínter y respuesta patológica en aproximadamente la mitad de los pacientes.

\section{Supervivencia en adenocarcinoma gástrico posterior a tratamiento adyuvante tratado en el Instituto Nacional de Cancerología de Colombia durante 2009}

\section{Fabio Grosso', Ricardo Brugés ${ }^{1}$, Licet Villamizar²}

${ }^{1}$ Grupo de Oncología Clínica, Instituto Nacional de Cancerología, Bogotá (Colombia).

2 Grupo de Investigación Clínica, Instituto Nacional de Cancerología, Bogotá (Colombia).

Objetivo. El objetivo de este estudio retrospectivo es determinar la supervivencia libre de enfermedad y global a dos años en pacientes con cáncer gástrico que recibieron tratamiento adyuvante en el Instituto Nacional de Cancerología de Colombia (INC) durante 2009.

Pacientes y métodos. De forma retrospectiva se incluyeron pacientes con diagnóstico de adenocarcinoma gástrico o de la unión gastroesofágica que hubieran recibido quimioterapia adyuvante en el INC durante 2009. Se describieron las características clínicas, epidemiológicas, tratamiento recibido, supervivencia global y libre de enfermedad a dos años.

Resultados. Se incluyeron en el análisis 32 pacientes. La edad media fue de 55 años. Los hombres contaron el 56,2\% de los casos. El subtipo histológico predominante fue el intestinal $(53,1 \%)$. El grado de invasión tumoral T3-T4 se presentó en el 81,2\% y compromiso ganglionar en el $84,3 \%$, con un $34,3 \%$ de los casos con siete o más ganglios comprometidos. El 96,8\% recibieron tratamiento adyuvante de acuerdo con el protocolo McDonald. El 75\% de los pacientes completaron el tratamiento. En el $12 \%$ de los casos se suspendió el tratamiento por toxicidad. El seguimiento medio de los pacientes fue de 37,8 meses. La supervivencia global y libre de enfermedad a dos años en pacientes que recibieron quimioterapia adyuvante fue del $85,18 \%$ y $77,4 \%$, respectivamente.

Conclusiones. Este estudio mostró una mayor supervivencia global y libre de enfermedad para pacientes que recibieron tratamiento adyuvante en el INC frente a la encontrada en los estudios INT-0116 y Magic; la supervivencia es similar a estudios con población asiática. 